\title{
2777 cultures on conjunctivitis (whose nature is not determined): epidemiological survey in the course of 7 years of observation
}

\author{
Franco Giardini', Romolo Protti ${ }^{2}$, Angelo Bay³, Giuseppe Grandi', Cristina Pollino', \\ Renata Murisciano', Andrea Grosso² \\ 'Laboratorio Analisi, Ospedale Oftalmico, Torino \\ ${ }^{2}$ Divisione di Traumatologia, Ospedale Oftalmico, Torino \\ ${ }^{3}$ Divisione Glaucomi, Ospedale Oftalmico, Torino
}

Key words: Conjunctivitis, Ocular antibiotics, Microbiological culture, Ocular flora, Eye infection.

\section{SUMMARY}

Our survey considers all pathogens isolated in patients with conjunctivitis (whose nature must be determined) from January 2000 to December 2007 in Ophthalmic Hospital of Turin. Overall we examined 2879 strains of pathogens from 12874 patients. Among the most important isolates, Staphylococcus aureus represented 32\%, while Streptococcus pneumoniae $16.6 \%$. The Streptococcaceae were 632 strains in total, with $21.9 \%$ while Moraxella genus with 269 cases represented $9.3 \%$.

About the sensitivity to the antibiotics for ocular topic use, our data evaluated Levofloxacin as the most effective in vitro, followed, at a distance, by Ampicillin, Chloramphenicol and Ofloxacin. According to the collected data, one patient out of 5 , affected by conjunctivitis (whose nature is to be determined) shows positive culture. Therefore it is very important to carry out always the cultural ocular test in order to provide the oculist with the right therapeutic indication.

Received May 19, 2008

Accepted July 24, 2008

\section{INTRODUZIONE}

La congiuntiva ed i suoi annessi sono di solito sterili alla nascita, ma rapidamente vengono colonizzati da batteri saprofiti che costituiscono così la normale flora batterica congiuntivo-corneale. La proliferazione dei batteri saprofiti è sotto lo stretto controllo di numerosi fattori antibatterici endogeni (lactoferrina, lisozima, betalisine, componenti del complemento).

Alterazioni sia locali (trichiasi, lagoftalmo, occhio secco, dacriocistiti) che sistemiche possono far diminuire i sistemi difensivi endogeni predisponendo alle infezioni congiuntivo-corneali.

Le congiuntiviti batteriche si possono suddividere, da un punto di vista clinico, in acute e croniche. Le congiuntiviti batteriche acute generalmente si manifestano con sensazione di corpo estraneo e lacrimazione, seguite da modesta o importante secrezione mucopurulenta ed iperemia (10) congiuntivale diffusa (9).

Le congiuntiviti batteriche croniche hanno segni clinici quali iperemia congiuntivale con follicoli e papille, secrezione minima ed ispessimento congiuntivale.

\section{MATERIALI E METODI}

In questo lavoro abbiamo considerato 12874 colture oculari da pazienti con sindrome congiuntivale di n.d.d. afferiti al nostro Pronto Soccorso o direttamente da prelievi effettuati sui pazienti da noi stessi in laboratorio, dal gennaio 2000 a tutto il dicembre 2007.

Di questi campioni abbiamo analizzato 2777 colture oculari positive per schizomiceti o miceti patogeni, ma in realtà i ceppi isolati sono in totale 2879 in quanto vi sono state 102 colture con flora mista. I pazienti di questa indagine random avevano un'età compresa tra 2 e 96 anni.

Parte di essi (29.7\%) era già in trattamento antibiotico locale.

Per i campioni oculari pervenuti a noi dal Pronto Soccorso sono stati usati tamponi con terreno di trasporto (Stuart); sui restanti pazienti, afferiti direttamente presso il nostro laboratorio (circa il

\section{Corresponding author: Franco Giardini}

Laboratorio Analisi, Ospedale Oftalmico

Via Juvarra 19 - 10122 Torino

Tel.: 0 I I-566603 I; Fax: 0 I I-5666I86 - E-mail: franco.giardini@libero.it 
$50 \%$ ), il prelievo è stato eseguito con tamponcini in calcio alginato (16) di piccolo calibro, imbibiti previamente in soluzione fisiologica sterile e passati nel fornice congiuntivale inferiore e/o superiore, dall'interno verso l'esterno, avendo cura di non toccare la rima palpebrale.

I terreni utilizzati sono quelli di routine: agar cioccolato (BD) (aerobiosi a $37^{\circ} \mathrm{C}$ per $48 \mathrm{~h}$ ), agar cioccolato più bacitracina (BD) (capnofilia fino a 48h), provettoni con brodo cuore-cervello e agar sabouraud dextrose (BD). Solo per i pazienti in età pediatrica si aggiungeva la semina su una piastra di agar sangue Columbia (BD).

Sono state considerate significative anche le colture che davano uno sviluppo di una o due colonie di schizomiceti patogeni, in quanto la flora oculare è sempre paucimicrobica.

Se dopo 48h di incubazione non vi era stata alcuna crescita microbica la coltura era considerata negativa.

\section{Identificazione batterica}

Riguardo all'identificazione degli schizomiceti isolati si è proceduto secondo le consuete tecniche di microbiologia basate sulle caratteristiche morfologiche delle colonie, sulle modalità della loro crescita in vitro, sulle loro capacità fermentative e sulle caratteristiche biochimiche.

In particolare per ciò che concerne l'identificazione degli schizomiceti Gram negativi non fermentanti è stato usato il metodo API NE (bioMérieux).

\section{Antibiogrammi}

Gli antibiogrammi da noi effettuati di routine sulle infezioni congiuntivali di n.d.d., non essendo prevista una terapia sistemica collaterale, a differenza del nostro protocollo nei confronti delle infezioni oculari gravi a prognosi severa, prevedevano solo l'utilizzo degli antibiotici presenti nei presidi farmaceutici per uso topico oculare (9).

Gli antibiotici saggiati erano: Ampicillina, Tetraciclina, Cloramfenicolo, Neomicina, Amikacina, Gentamicina, Netilmicina, Tobramicina, Ac. Fusidico, Vancomicina, Ofloxacina, Norfloxacina, Lomefloxacina, Ciprofloxacina, Levofloxacina.

Gli antibiogrammi erano effettuati con il metodo Kirby-Bauer per il quale venivano utilizzate piastre di Agar M. Hinton, e dischetti standard (Becton Dickinson) imbibiti di antibiotico.

\section{RISULTATI}

Analizzando questi dati si evince che $S$. aureus è, tra i patogeni isolati, al primo posto (898 casi) sfiorando il 32\% dei patogeni isolati, seguono le
Streptococcaceae con 632 casi totali (21.9\%) e lo Streptococcus pneumoniae (8) con 479 casi (16.6\%).

Tra i non fermentanti vediamo il genere Moraxella (10) responsabile di congiuntivi angolari, con 269 casi (9.3\%) e il genere Pasteurella con 100 casi.

Le Pseudomonadaceae con 152 casi in totale (5.3\%) sono risultate frequentemente multiresistenti, così come il genere Serratia (101 casi, pari $3.5 \%$ ) che supporta congiuntiviti spesso ad andamento clinico rapidamente invasivo.

Il genere Haemophilus (94 casi, pari al 3.2\% e al $18.1 \%$ delle sole infezioni infantili) nella quasi totalità dei casi concerneva infezioni oculari della prima infanzia (10) con percentuali di isolamento abbastanza in linea con molti dati di letteratura per quella fascia d'età (attorno al 22-23\%)(8). Mentre il genere Pasteurella (100 casi) di norma aveva un profilo di antibiotico-resistenza piuttosto basso.

Ben 791 ceppi (27.5\%) tra quelli isolati nelle colture oculari presentavano resistenza ad almeno 10 antibiotici sui 14 da noi saggiati.

I miceti in queste patologie congiuntivali non sono frequenti e anche in questo lavoro, sono risultati essere l’1.1\% degli isolamenti (2).

\section{Considerazioni sulla flora batterica isolata}

S. aureus: Uno dei motivi che possono determinare la prevalenza di questo microbiota (12) sugli altri è da rilevarsi nell' ambiente particolarmente salino che le lacrime creano nella congiuntiva, favorendo certamente la crescita di schizomiceti alofili quali Staphylococcus.

S. pneumoniae: un ruolo certamente di rilievo lo riveste $S$. pneumoniae con l'isolamento di 479 ceppi (16.6\%). L'alta frequenza con cui viene isolato questo patogeno è dovuta anche ad una buona percentuale di pazienti in età pediatrica $(8$, $10,16)$, tra i quali, è risaputo, lo Streptococcus pneumoniae è uno degli schizomiceti più frequentemente presenti sia a livello oculare che in ambito ORL.

Gram negativi "difficili” non fermentanti: Questi batteri non sempre in batteriologia oculare vengono considerati nel modo dovuto, al più, quasi sempre, sono considerati dei saprofiti e hanno elevate capacità di adattarsi fenotipicamente all'ambiente. Nelle infezioni congiuntivali oculari alcuni di essi (Moraxella, meglio nota come bacillo di Morax-Axenfeld) rivestono invece il ruolo di patogeni e l'interessante percentuale da noi rilevata (12.8\%), unitamente a quadri di discreta antibiotico-resistenza (specie per il genere Moraxella)(7, 11) sovente espressi da questo gruppo di batteri, li rende degni della dovuta attenzione oltrechè in oftalmologia 
anche in batteriologia oculare.

CoNS: Lo Staphylococcus coagulasi-negativo sebbene isolato in questo lavoro solo in 73 casi (2.5\%), anche in batteriologia oculare rivela notevoli profili di elevata antibiotico-resistenza e da qualche anno compare anche tra i responsabili di talune affezioni oculari a prognosi severa quali gli ascessi.

Genere Serratia: Tra le Enterobacteriaceae prevale il genere Serratia che associa alla sua attività proteolitica anche una certa antibiotico - resistenza.

Pseudomonadaceae: Questi batteri normalmente sono responsabili di gravi infezioni oculari non congiuntivali, e ciò spiega la bassa percentuale (152 casi pari al 5.3\%) di isolamenti in colture da infezioni della congiuntiva (13).

Streptococcaceae: Di questi batteri isolati (Streptococchi alfa e beta emolitici) (7) va sottolineata la notevole e spiccata antibiotico resistenza che li rende, a livello oculare, tra i più ribelli alle cure antibatteriche se non mirate. In particolare sono stati riscontrati e ritenuti importanti dal punto di vista clinico gli Streptococchi viridanti (alpha emolitici) $(6,15)$ isolati che, secondo l'esperienza del nostro ospedale monospecialistico, debbono esser trattati con estrema attenzione per non rischiare di cronicizzare l'affezione congiuntivale da essi sostenuta. Negli ultimi 3 anni della nostra casisistica inoltre abbiamo riscontrato alcune infezioni oculari a prognosi severa (endoftalmiti, ascessi) sostenute da Streptococco alpha emolitico in coltura pura.

In tabella 1 e 2 sono illustrati i risultati delle indagini colturali ed in tabella 3 sono presentati i dati di sensibilità dei singoli ceppi batterici e gruppi batterici ad alcuni antibiotici per uso topico oculare.
Tabella I. Patogeni isolati ripartiti per ceppi

\begin{tabular}{lc}
\hline Staphylococcus aureus & 898 \\
\hline MRSA * & 10 \\
\hline CoNS ** & 73 \\
\hline Streptococcus alpha - haemolyticus & 592 \\
\hline Streptococcus beta - haemolyticus & 40 \\
\hline Streptococcus pneumoniae & 479 \\
\hline Moraxella spp. & 269 \\
\hline Pasteurella spp. & 100 \\
\hline Pseudomonas aeruginosa & 144 \\
\hline Pseudomonas putida & 4 \\
\hline Burkholderia cepacia & 4 \\
\hline Klebsiella oxytoca & 20 \\
\hline Klebsiella pneumoniae & 7 \\
\hline Serratia marcescens & 73 \\
\hline Serratia liquefaciens & 28 \\
\hline Proteus mirabilis & 7 \\
\hline Citrobacter amalonaticus & 4 \\
\hline Citrobacter diversus & 2 \\
\hline Enterobacter agglomerans & 6 \\
\hline Haemophilus influenzae & 81 \\
\hline Haemophilus parainfluenzae & 13 \\
\hline * MRSA= Stafilococco aureo meticillino-resistente \\
** CoNS= Stafilococco coagulasi negativo \\
\hline
\end{tabular}

Tabella 2. Miceti isolati ripartiti per ceppi

\begin{tabular}{ll}
\hline Candida albicans & 8 \\
\hline Candida krusei & 2 \\
\hline Candida lusitaniae & $\mathrm{I}$ \\
\hline Aspergillus fumigatus & 2 \\
\hline Aspergillus niger & 8 \\
\hline Penicillium spp & $\mathrm{I}$ \\
\hline Mucor spp & $\mathrm{I}$ \\
\hline Fusarium spp & $\mathrm{I}$ \\
\hline Blastomyces spp. & $\mathrm{I}$
\end{tabular}

Tabella 3. Sensibilità dei singoli ceppi batterici e gruppi batterici ad alcuni antibiotici per uso topico oculare

\begin{tabular}{lcccccccccc}
\hline Microorganismi & Casi & AMP. & CLOR. & NET. & OFL. & TOB. & NORF. & LOM. & LEV. & AC.FUS. \\
\hline S. aureus & 898 & $21.7 \%$ & $68.5 \%$ & $92.1 \%$ & $71.9 \%$ & $66.5 \%$ & $61.6 \%$ & $74.9 \%$ & $88.7 \%$ & $86.4 \%$ \\
\hline S. pneumoniae & 479 & $95.7 \%$ & $94.8 \%$ & $23.3 \%$ & $78.4 \%$ & $3.6 \%$ & $47.4 \%$ & $46.5 \%$ & $93.2 \%$ & $20.7 \%$ \\
\hline Strepto $\alpha$ e B-emol. & 632 & $83.8 \%$ & $97.2 \%$ & $14.0 \%$ & $67.6 \%$ & $3.4 \%$ & $14.5 \%$ & $20.1 \%$ & $95.0 \%$ & $12.8 \%$ \\
\hline Haemophilus spp. & 94 & $50.0 \%$ & $100 \%$ & $79.2 \%$ & $95.8 \%$ & $25.0 \%$ & $100 \%$ & $100 \%$ & $100 \%$ & $5.8 \%$ \\
\hline Serratia spp. & 101 & $11.3 \%$ & $62.2 \%$ & $80.0 \%$ & $88.9 \%$ & $53.3 \%$ & $77.8 \%$ & $84.5 \%$ & $91.1 \%$ & $0.0 \%$ \\
\hline Pseudomonas spp. & 152 & $11.1 \%$ & $22.2 \%$ & $44.4 \%$ & $77.8 \%$ & $38.9 \%$ & $50.0 \%$ & $88.9 \%$ & $100 \%$ & $13.3 \%$ \\
\hline Congiuntiviti (tutte) & 2854 & $50.0 \%$ & $76.6 \%$ & $59.2 \%$ & $79.9 \%$ & $38.3 \%$ & $56.5 \%$ & $63.6 \%$ & $91.0 \%$ & $66.4 \%$ \\
\hline
\end{tabular}

\section{Nota.}

a) Per non appesantire, a nostro avviso, in modo esagerato la tabella abbiamo ritenuto opportuno prendere in esame per questo lavoro solo l'attività in vitro di alcuni antibiotici per uso topico oculare $(8,14)$ versus alcuni ceppi isolati, e ne abbiamo considerata solo la sensibilità.

In particolare in questo lavoro vengono considerati i seguenti antibiotici: Ampicillina, Cloramfenicolo, Netilmicina, Ofloxacina, Tobramicina, Norfloxacina, Lomefloxacina, Levofloxacina, Ac. Fusidico.

b) Al fondo della tabella abbiamo voluto riassumere tutte le congiuntiviti, causate da tutti gli schizomiceti da noi isolati, per dare al lettore un profilo di massima della sensibilità ai vari antibiotici. 


\section{CONCLUSIONI}

I dati raccolti presso il laboratorio Analisi dell'Ospedale Oftalmico parlano di 12874 pazienti con congiuntiviti di n.d.d., di cui l'89.6\% erano adulti. Delle colture effettuate a seguito degli strisci congiuntivali eseguiti su ciascun paziente ben 2777 hanno dato esito colturale positivo per schizomiceti o miceti patogeni, risultando perciò che circa 1 paziente su 5 presentava coltura oculare positiva.

Occorre a questo punto tenere presente che lo striscio congiuntivale veniva eseguito solo se il paziente aveva sospeso un'eventuale antibioticoterapia topica da almeno $48 \mathrm{~h}$, ma che,secondo la nostra pluridecennale esperienza, molte delle persone che si presentano al nostro ospedale per sottoporsi a questo esame volutamente non asseriscono il vero.

Può sembrare un controsenso, ma sappiamo che alcuni pazienti che ci chiedevano spiegazioni di un referto con esito colturale negativo ammettevano tardivamente, in quella circostanza, di aver instillato colliri antibiotici o pomate oftalmiche magari la mattina stessa dell'esame. E di norma sostenevano di non aver ben capito le istruzioni dell'oculista, le nostre domande all'atto dell'esame,o di temere un peggioramento delle proprie condizioni soggettive nel caso di una sospensione della terapia che stavano effettuando. Per non parlare poi di molti pediatri che, dopo aver trattato il bambino con diverse molecole, alla fine si arrendono ed inviano il loro piccolo paziente a fare l'esame sovente con ancora in corso la terapia antibiotica congiuntivale.

Tutto questo ci porta a concludere che certamente il numero delle colture positive per flora microbica patogena sarebbe superiore se l'esame dello striscio oculare venisse fatto a soggetti che hanno realmente sospeso ogni eventuale terapia.

In ogni caso la percentuale di pazienti con coltura oculare positiva anche in queste condizioni ci consente di affermare che la coltura congiuntivale è non solo importante ma soprattutto doverosamente prescrivibile, proprio per l'alto numero di patogeni isolati e per poter fornire all'oculista l'indicazione clinica, talora indispensabile, di un antibiogramma mirato con antibiotici per uso topico oculare.

Per ciò che concerne gli isolamenti colturali che risultano da questa osservazione pluriennale possiamo anzitutto dire che anche nella nostra statistica epidemiologica $S$. aureus detiene il primo posto con 898 ceppi isolati. Questi dati sono in linea con la statistica eziologia ed epidemiologica della patologia congiuntivale di n.d.d. a livello internazionale.
Interessante la presenza significativa di 73 casi di CoNS anche nella patologia da congiuntivite, mentre ci preme sottolineare l'alto numero di infezioni congiuntivali da Streptococcus alpha haemolyticus (592 casi su 632 totali di Streptococcaceae) $(6,16)$.

$\mathrm{E}$ a questo proposito è necessario evidenziare l'importanza della semina routinaria, per ciascun paziente, di un provettone di brodo cuore-cervello.

Infatti, quando Streptococcus alpha haemolyticus è il responsabile di una congiuntivite,quasi sempre, per isolarlo, non sono sufficienti i terreni solidi ed è indispensabile il brodo cuore-cervello.

Occorre sempre tener presente che, oltre che per l'azione dei residui di farmaci antibiotati in situ, sovente la carica batterica è molto bassa poiché la flora oculare eventualmente presente è sempre paucimicrobica.

Quanto all'attività antibatterica in vitro nei confronti dei patogeni isolati dalle colture congiuntivali, dalla tabella 3 che raggruppa tutti i casi di congiuntiviti positivi solo per batteri, possiamo avere delle indicazioni di massima.

In particolare notiamo l'ottima risposta che ha dato e dà, in vitro, la Levofloxacina verso un po' tutti i ceppi batterici isolati (91.0\%)(15).

Per contro la peggior sensibilità è di Tobramicina $(3,5,9)$ con il 38.3\% efficacia.

Anche l'Ofloxacina ed il Cloramfenicolo (1), sebbene, per così dire, antibiotici di lungo corso, con quasi il 77\% di sensibilità danno sicure garanzie per eventuali terapie alla cieca, pro tempore, in attesa di dati specifici dal microbiologo.

Andando poi nello specifico dei vari schizomiceti patogeni qui esaminati possiamo sottolineare l'86.1\% di ceppi di S. aureus sensibili all'Acido Fusidico, particolarmente indicato nei confronti di questo microbiota (14), e il 92.1\% di ceppi sensibili alla Netilmicina.

Considerando gli Streptococchi in genere, e quindi anche S. pneumoniae, si evince che il quadro di sensibilità è tra loro abbastanza sovrapponibile. Ottime le risposte ad Ampicillina, Cloramfenicolo e Levofloxacina (10) con valori nettamente oltre il $90 \%$ di sensibilità. Trascurabili le sensibilità a Tobramicina e Netilmicina.

Se poi poniamo l'attenzione sul genere Haemophilus e su S. pneumoniae, schizomiceti che sostengono infezioni congiuntivali a carico specialmente della fascia d'età infantile, valgono molte delle considerazioni suesposte con ottime percentuali di sensibilità di Cloramfenicolo e dei Fluorochinoloni (specie per Haemophilus). Scarsissima la sensibilità di Tobramicina e Acido Fusidico. 
Circa Serratia e le Pseudomonadaceae, che esprimono talora ceppi altamente aggressivi nei confronti dei tessuti oculari, possiamo rilevare che anche in questi casi la Levofloxacina risulta essere l'antibiotico più efficace in vitro.

Tutte queste considerazioni, magari ponderate in modo sinottico, ci portano a ripetere che per lo specialista è indispensabile avere in supporto dalla Microbiologia un antibiogramma mirato (on antibatterici per uso topico e non solo) al fine di risolvere rapidamente la patologia congiuntivale (è bene ricordare che le congiuntiviti causano ogni anno assenze dal lavoro per migliaia di giornate e sono talora temporaneamente invalidanti) e di non correre il rischio di cronicizzarla o di aumentare le antibiotico-resistenze.

Il significativo numero di casi affrontati dal nostro ospedale monospecialistico rende questo lavoro un monitoraggio epidemiologico e di antibioticoresistenza riferito alle congiuntiviti, almeno per la nostra area geografica. E i nostri dati vengono messi a conoscenza soprattutto dell'oculista anche per fornirgli delle indicazioni attendibili per la terapia praticata in attesa del responso di laboratorio.

\section{BIBLIOGRAFIA}

1. Agius - Fernandez A, Patterson A, Fsadni M, Jauch A, Raj PS. Topical Lomefloxacin versus topical Chloramphenicol in the treatment of acute bacterial conjunctivitis. Clin Drug Investig 1998; 15 (4): 263-9.

2. Ainley R, Smith B. Fungal flora of the conjunctival sac in healthy and diseased eyes. Br J Ophthalm 1965; 49: 505-515.

3. Chalita MR, Hofling - Lima AL, Paranhos A, Schor P, Belfort R. Shifting trends in vitro antibiotic susceptibilities for common ocular isolated during a period of 15 years. Am J Ophthalmol 2004; 137: 43-51.

4. Chung CW. Eye disorders: Bacterial conjunctivitis.
Clinical Evidence 2000; 3: 305-310.

5. Everett SL, Kowalski RP, Karenchak LM, Landsittel $\mathrm{D}$, Day R, Gordon YJ. An in vitro comparison of the susceptibilities of bacterial isolates from patients with conjunctivitis and blepharitis to newer and established topical antibiotics. Cornea 1995; 14: 382-387.

6. Gerkowicz M, Koziol-Montewka $M$, et al. Identification of bacterial flora of conjunctival sac in congenital nasolacrimal duct obstruction in children. Klin Oczna, 2005; 107 (1-3): 83.

7. Giardini F, Attisano C, Vana M, Grandi G, Hassan A. Epidemiologia e antibiotico-resistenza in 150 casi di infezioni batteriche oculari in età pediatrica. Farmacologia Oculare. Atti Congresso Naz Sanremo 1996; 23-30.

8. Gigliotti F, Hendley JO, Morgan J, Michaels R, Dickens M, Lohr J. Efficacy of topical antibiotic therapy in acute conjunctivitis in children. Am $J$ of Pediatrics 1984; 104: 623-626.

9. Hovding G. Acute bacterial conjunctivitis. Acta Ophthalmol 2008; 86 (1): 2-4.

10. Kowalski RP, Dhaliwal DK. Ocular bacterial infections: current and future treatment options. Expert Rev. Anti Infect Ther 2005; 2(1): 131-139.

11. Limberg MB. A review of bacterial keratitis and bacterial conjunctivitis. Am J Opht 1991; 112: 28-98.

12. Miller B, Ellis PP. Conjunctival flora in patients receiving immunoppressive drugs. Arch Ophthalm 1977; 95: 2012-2014.

13. Pinna A, Usai D, Sechi LA, Molicotti P, Zanetti S, Carta A. Detection of virulence factors in Pseudomonas aeruginosa strains isolated from contact lens associated corneal ulcers. Cornea 2008; 27(3): 320-326.

14. Rietveld RP, Riet G, Bindels PJ, Bink D, Sloos JH, Vaan Meert HC. The treatment of acute infectious conjunctivitis with fusidic acid: a randomised controlled trial. Br J Gen Pact 2005; 55(521): 924-30.

15. Rose P. Topical antibiotics for acute bacterial conjunctivitis. Expert Opin Pharmacother 2007; 8(12): 1903-21.

16. Tarabishy AB, Hall GS, ProcopGW, Jeng BH. Bacterial culture isolates from hospitalized patients with conjunctivitis. AM J Ophthalmol 2006; 142(4): 678-80.

17. Wilson LA, Sexton RR. Laboratory aids in diagnosis. Clinical Ophthalm 1982; 4: 1-15. 